
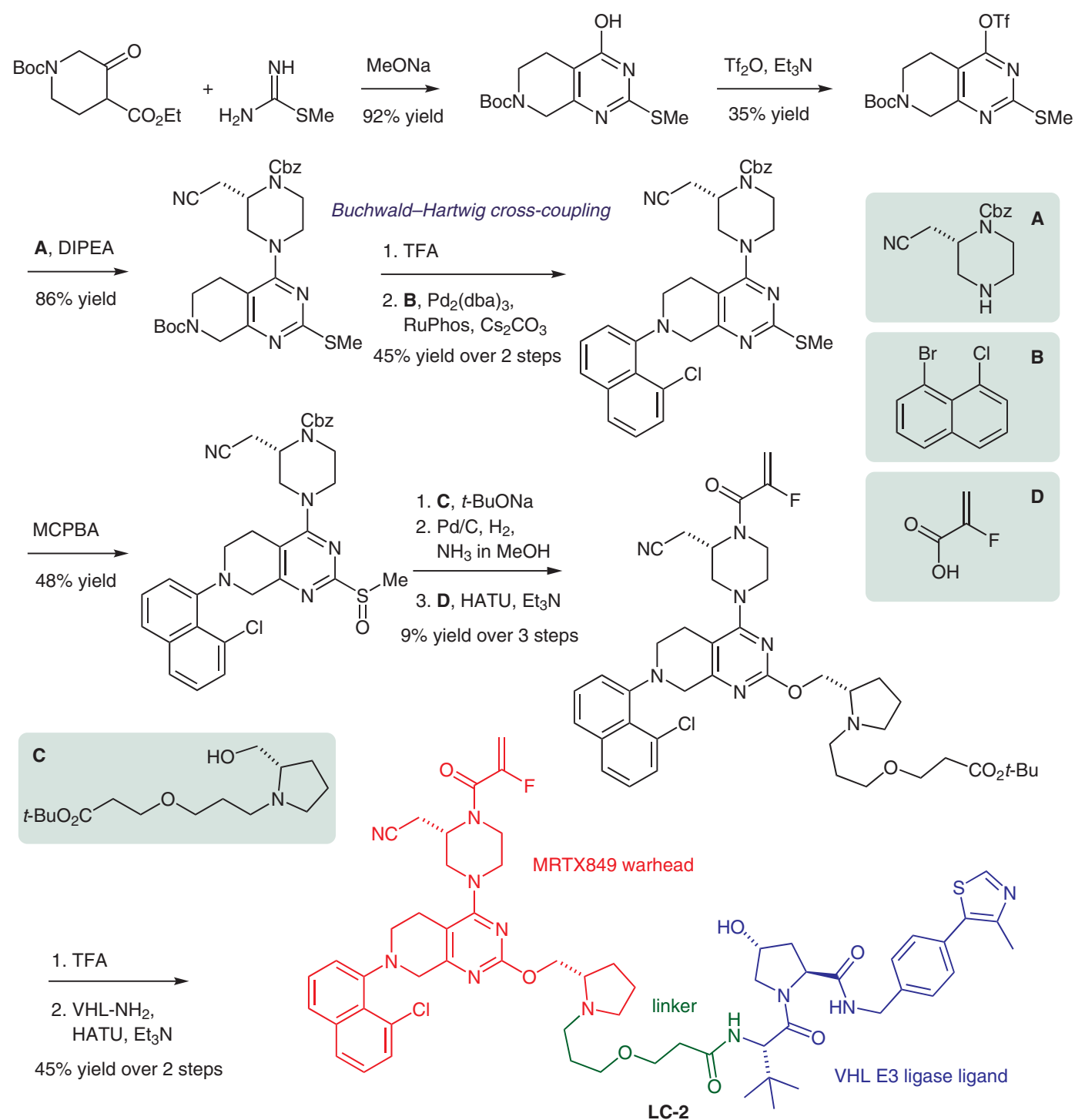

Chemistry in

Medicine and

Biology

Key words

\title{
KRAS
}

targeted

degradation

cancer

drug development

\section{Syniact \\ of the}

Significance: KRas is one of the most promising cancer drug targets, but targeting it has been a challenge. Crews and co-workers employed covalent KRas G12C inhibitors for the development of PROTACS that allowed for its targeted degradation.
Comment: A solvent-exposed site in the clinical drug candidate MRTX849 was identified and used for conjugation to a linker and a VHL ligand to yield the PROTAC LC-2. 